\title{
Optically detected magnetic resonance studies of point defects in quaternary GaNAsP epilayers grown by vapor phase epitaxy
}

Daniel Dagnelund, Jan E. Stehr, A Yu Egorov, Weimin Chen and Irina Buyanova

\section{Linköping University Post Print}

N.B.: When citing this work, cite the original article.

Original Publication:

Daniel Dagnelund, Jan E. Stehr, A Yu Egorov, Weimin Chen and Irina Buyanova, Optically detected magnetic resonance studies of point defects in quaternary GaNAsP epilayers grown by vapor phase epitaxy, 2013, Applied Physics Letters, (102), 2, .

http://dx.doi.org/10.1063/1.4781459

Copyright: American Institute of Physics (AIP) http://www.aip.org/

Postprint available at: Linköping University Electronic Press http://urn.kb.se/resolve?urn=urn:nbn:se:liu:diva-89522 


\section{AIP Applied Physics Letters}

\section{Optically detected magnetic resonance studies of point defects in quaternary GaNAsP epilayers grown by vapor phase epitaxy}

D. Dagnelund, Jan Stehr, A. Yu. Egorov, W. M. Chen, and I. A. Buyanova

Citation: Appl. Phys. Lett. 102, 021910 (2013); doi: 10.1063/1.4781459

View online: http://dx.doi.org/10.1063/1.4781459

View Table of Contents: http://apl.aip.org/resource/1/APPLAB/v102/i2

Published by the American Institute of Physics.

\section{Related Articles}

Insertion of lithium ions into $\mathrm{TiO} 2$ (rutile) crystals: An electron paramagnetic resonance study of the Li-associated Ti3+ small polaron

J. Appl. Phys. 113, 053712 (2013)

Acceptors in $\mathrm{ZnO}$ nanocrystals: A reinterpretation

Appl. Phys. Lett. 101, 262106 (2012)

Electron paramagnetic resonance probed oxygen deficiency in SrTiO3 with different cap layers

J. Appl. Phys. 112, 123720 (2012)

Electron spin resonance features of the $\mathrm{Ge} \mathrm{Pb1}$ dangling bond defect in condensation-grown

(100)Si/SiO2/Si1-xGex/SiO2 heterostructures

J. Appl. Phys. 112, 074501 (2012)

Electron spin resonance signature of the oxygen vacancy in $\mathrm{HfO} 2$

Appl. Phys. Lett. 101, 102904 (2012)

\section{Additional information on Appl. Phys. Lett.}

Journal Homepage: http://apl.aip.org/

Journal Information: http://apl.aip.org/about/about_the_journal

Top downloads: http://apl.aip.org/features/most_downloaded

Information for Authors: http://apl.aip.org/authors

\section{ADVERTISEMENT}

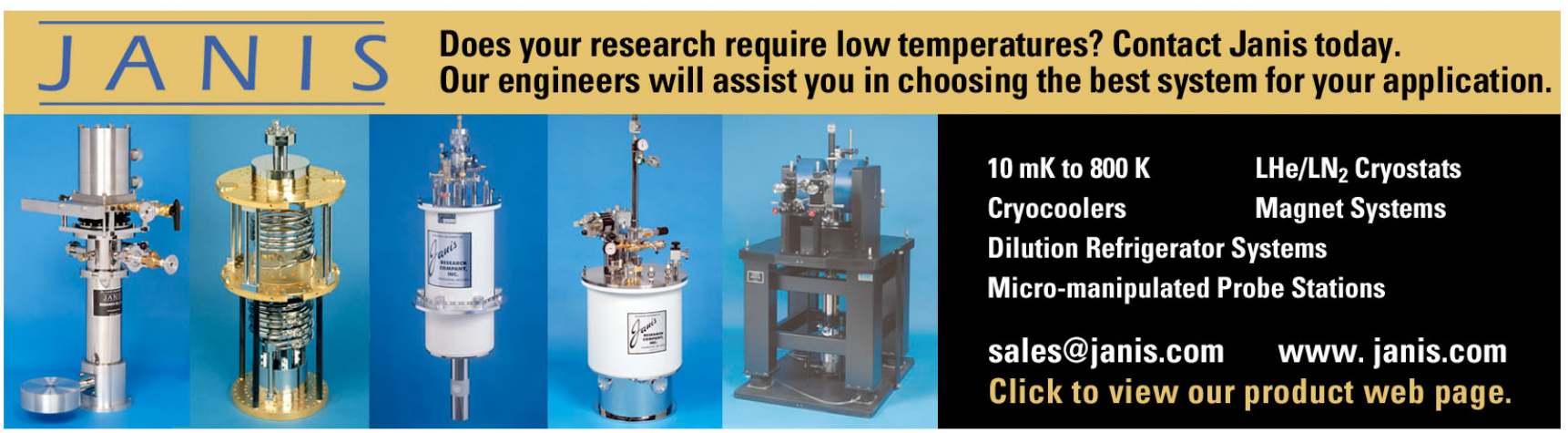




\title{
Optically detected magnetic resonance studies of point defects in quaternary GaNAsP epilayers grown by vapor phase epitaxy
}

\author{
D. Dagnelund, ${ }^{1}$ Jan Stehr, ${ }^{1}$ A. Yu. Egorov, ${ }^{2,3}$ W. M. Chen, ${ }^{1}$ and I. A. Buyanova ${ }^{1}$ \\ ${ }^{1}$ Department of Physics, Chemistry and Biology, Linköping University, S-581 83 Linköping, Sweden \\ ${ }^{2}$ Nanotechnology Center for Research and Education, St. Petersburg Academic University, \\ Russian Academy of Sciences, St. Petersburg 195 220, Russia \\ ${ }^{3}$ Ioffe Physical Technical Institute, Russian Academy of Sciences, St. Petersburg 194 021, Russia
}

(Received 27 November 2012; accepted 4 January 2013; published online 17 January 2013)

\begin{abstract}
Defect properties of quaternary GaNAsP/GaP epilayers grown by vapor phase epitaxy (VPE) are studied by photoluminescence and optically detected magnetic resonance techniques. Incorporation of more than $0.6 \%$ of nitrogen is found to facilitate formation of several paramagnetic defects which act as competing carrier recombination centers. One of the defects (labeled as $\mathrm{Ga}_{\mathrm{i}}-\mathrm{D}$ ) is identified as a complex defect that has a $\mathrm{Ga}$ interstitial $\left(\mathrm{Ga}_{\mathrm{i}}\right)$ atom residing inside a $\mathrm{Ga}$ tetrahedron as its core. A comparison of $\mathrm{Ga}_{\mathrm{i}}-\mathrm{D}$ with other $\mathrm{Ga}_{\mathrm{i}}$-related defects known in ternary GaNP and GaNAs alloys suggests that this defect configuration is specific to VPE-grown dilute nitrides. (C) 2013 American Institute of Physics. [http://dx.doi.org/10.1063/1.4781459]
\end{abstract}

Epitaxial III-V semiconductor light sources on silicon would tremendously increase the functionality of Si microelectronics and are promising for the realization of optoelectronic integrated circuits. For solar cell applications, Si-based multi-junction stacking would improve efficiency and reduce cost relative to conventional $\mathrm{Si}$ and III-V multijunction cells. Direct epitaxial growth of conventional direct-band-gap III-V compounds (such as GaAs or InP) on $\mathrm{Si}$ is probably the most straightforward approach. However, due to a large lattice mismatch, high densities of threading or misfit dislocations and also point defects are formed in the III-V epitaxial films directly grown on Si substrates, preventing achievement of satisfactory performance. ${ }^{1}$ An exciting approach to circumvent this problem is epitaxial growth of compound semiconductors that are lattice matched to $\mathrm{Si}$, such as the $\mathrm{GaN}_{\mathrm{x}} \mathrm{As}_{\mathrm{y}} \mathrm{P}_{1-\mathrm{x}-\mathrm{y}}$ quaternary alloy.

GaNAsP belongs to an interesting class of dilute nitrides that have recently attracted great attention owing to their fascinating physical properties. In dilute nitrides, the replacement of a small fraction $(x \sim 1 \%)$ of phosphorus or arsenic atoms by nitrogen atoms causes highly nonlinear effects in the electronic properties of the host lattice. ${ }^{2,3}$ The pronounced effect of $\mathrm{N}$ on the band structure of $\mathrm{GaP}$ leads to a giant reduction in the bandgap energy and the $\mathrm{N}$-induced crossover from an indirect bandgap in GaP to a quasi-direct bandgap in GaNP. ${ }^{4,5}$ This renders this material as having a high potential for visible light emitting diodes, multi-junction solar cells, heterojunction bipolar transistors, and terahertz applications. Unfortunately, epitaxial growth of dilute nitrides remains a great challenge. The required non-equilibrium growth conditions together with the disparity between $\mathrm{N}$ and the replaced group- $\mathrm{V}$ atoms are known to favor formation of various grown-in defects, which may give rise to deep levels in the gap and are considered to play a major role in limiting the optical quality of alloys. In fact, the issue of point defects is one of the main problems we are currently facing that hinders a rapid progress of dilute nitrides for various device applications in optoelectronics and photonics. Therefore, detailed knowledge about nature and formation mechanisms of defects and their influence on physical properties of alloys is necessary in order to control them. ${ }^{6}$ Recently, we have demonstrated that $\mathrm{Ga}$ interstitial $\left(\mathrm{Ga}_{\mathrm{i}}\right)_{-}$ related defects are the dominant grown-in defects formed during molecular beam epitaxy (MBE) growth of both $\mathrm{GaP}^{7,8}$ and $\mathrm{GaAs}^{9}$ — based dilute nitrides. However, chemical identification of point defects in quaternary GaNAsP alloys is currently still lacking. The aims of the present work are: (a) to study and identify important grown-in defects in $\mathrm{GaN}_{\mathrm{x}} \mathrm{As}_{\mathrm{y}} \mathrm{P}_{1-\mathrm{x}-\mathrm{y}}$, (b) to obtain information about the role of defects in carrier recombination processes, and finally, (c) to evaluate the obtained results in light of previous defects studies in dilute nitrides. Photoluminescence (PL) and optically detected magnetic resonance (ODMR) techniques will be employed for these purposes.

Quaternary $\mathrm{GaN}_{\mathrm{x}} \mathrm{As}_{\mathrm{y}} \mathrm{P}_{1-\mathrm{x}-\mathrm{y}}$ epilayers used for this study were grown by vapor-phase epitaxy (VPE) on GaP substrates with a nearly (001) crystallographic orientation. Dimetylhydrazine, $\mathrm{C}_{2} \mathrm{H}_{8} \mathrm{~N}_{2}$, was used as the source of nitrogen. The epilayers were grown on top of a $400 \mathrm{~nm}$-thick GaP buffer layer and at the substrate temperature in the range $610-650{ }^{\circ} \mathrm{C}^{10}$ They were capped by $200-400 \mathrm{~nm}$ thick GaP layer. The most important parameters of the samples are summarized in Table I. PL and ODMR measurements were preformed at $5 \mathrm{~K}$ using either the $532 \mathrm{~nm}$ line of a solid state laser or the $590 \mathrm{~nm}$ line of a dye laser as an excitation source. PL signals were dispersed by a $0.4 \mathrm{~m}$ single grating monochromator. A charge coupled device camera or a Si photodiode were

TABLE I. Parameters of the GaNAsP epilayers studied in this work.

\begin{tabular}{lccc}
\hline \hline Sample No. & {$[\mathrm{N}](\%)$} & {$[$ As] $(\%)$} & Thickness $(\mathrm{nm})$ \\
\hline$\# 15$ & 0.6 & 0 & 100 \\
$\# 5$ & 0.6 & 5 & 100 \\
$\# 14$ & 0.6 & 11.5 & 400 \\
$\# 11$ & 0.6 & 18 & 400 \\
$\# 13$ & 0.9 & 5 & 400 \\
$\# 10$ & 0.9 & 11.5 & 400 \\
$\# 12$ & 1.2 & 5 & 400 \\
$\# 9$ & 1.2 & 18 & 400 \\
\hline \hline
\end{tabular}


used for PL detection in the visible spectral range. ODMR signals were measured at X-band $(9212 \mathrm{MHz})$ and Q-band (33948 MHz) as spin-resonance induced changes of the PL intensity (detected by a Si photodiode) utilizing the lock-in technique in phase with an amplitude modulated microwave field at a frequency of $3 \mathrm{kHz}$.

Figure 1(a) shows effects of $\mathrm{N}$ and As incorporation on $5 \mathrm{~K}$ PL spectra of the GaNAsP alloys within the visible spectral range, which are dominated by excitonic emissions at $\mathrm{N}$-related localized states. ${ }^{11}$ Incorporation of $\mathrm{N}$ and As induces a monotonic redshift in the peak position of these emissions reflecting a reduction in the alloy bandgap energy. In addition to the redshift, incorporation of both $\mathrm{N}$ and As also causes a strong decrease in the near-bandgap emission intensity. Such decrease is often caused by formation of defects during the growth that act as centers of competing carrier recombination. In order to study and identify these defects, detailed ODMR studies were carried out.

Typical ODMR spectra obtained by monitoring the excitonic emissions are presented in Fig. 1(b), as function of $\mathrm{N}$ and As compositions. Incorporation of $\mathrm{N}$ caused an appearance of several ODMR signals that were, however, somewhat quenched in the As-containing alloys. All observed ODMR signals are negative, i.e., correspond to a decrease in the nearband-edge PL intensity (they are shown as positive in Fig. 1(b)

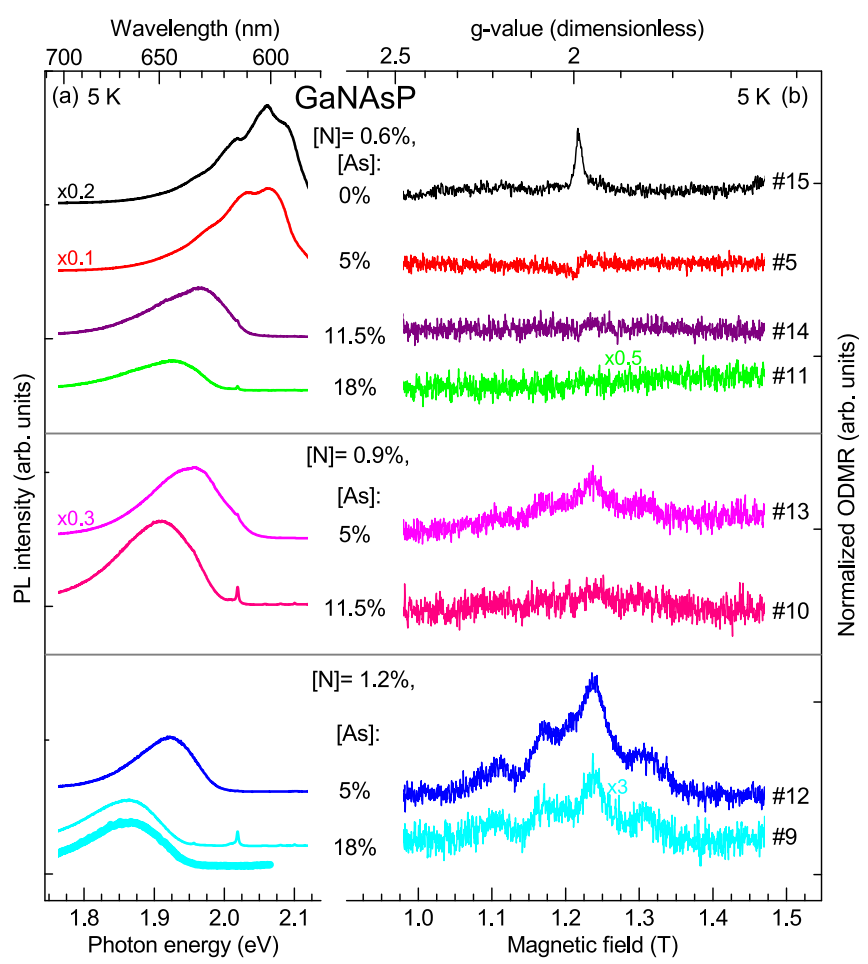

FIG. 1. (a) Representative PL spectra measured from the studied GaNAsP epilayers. All spectra except for the lowest one are taken with the excitation photon energy exceeding the GaP bandgap. A sharp PL feature at $2.02 \mathrm{eV}$ originates from the $\mathrm{GaP}$ substrate as it is not observed under below $\mathrm{GaP}$ bandgap excitation (see the lowest spectrum). No PL related to the GaNAsP epilayer was observed in near-infrared spectral range. (b) Isotropic ODMR spectra obtained at Q-band (33948 MHz) by monitoring the PL emissions shown in (a). The direction of the applied magnetic field is parallel to the [011] crystallographic direction and the ODMR intensity is normalized to the PL intensity. The ODMR signals are negative and are shown as positive in (b) for easy viewing. All ODMR spectra except the topmost one are taken with the excitation photon energy of $2.10 \mathrm{eV}$. The ODMR spectra from the sample $\# 15$ is taken under excitation at $2.33 \mathrm{eV}$. All data were obtained at $5 \mathrm{~K}$. merely for easy viewing). Before discussing effects of $\mathrm{N}$ and As incorporation on the defect formation, let us first provide a brief overview of the detected ODMR signals, which can be attributed to several different paramagnetic centers. The first signal consists of a single, and rather narrow line which is related to a paramagnetic center with an effective electron spin $S=1 / 2$ and $g$-value close to 2. It is detected only in the ternary GaNP with the lowest $\mathrm{N}$ content of $0.6 \%$ (sample \#15 in Fig. 1(b)). Positive identification of the corresponding defect is not possible, unfortunately, due to a lack of resolved hyperfine structure. For higher $\mathrm{N}$ compositions (samples \#13, \#10, \#12, and \#9), the ODMR spectra contain a rich pattern of lines spreading over a wide field range. The corresponding multiline ODMR spectra were analyzed using the spin Hamiltonian applicable for defects with an effective electronic spin $\mathrm{S}=1 / 2, H=\mu_{\mathrm{B}} g \mathbf{B} \cdot \mathbf{S}+A \mathbf{S} \cdot \mathbf{I}$. Here, $\mu_{\mathrm{B}}$ is the Bohr magneton, $\mathbf{B}$ is the external magnetic field, and $A$ is the central hyperfine parameter that describes coupling of the electron spin with a nuclear spin $\mathbf{I}$. The electronic gfactor and the $A$ parameter are scalars here, since all observed ODMR signals are isotropic. As shown in Fig. 2, the structure of the multiline ODMR spectra can be accurately reproduced by assuming a paramagnetic defect center with $S=1 / 2$ and a strong hyperfine interaction between the localized electron spin and the nuclear spin of a $\mathrm{Ga}$ atom $\left(60 \%{ }^{69} \mathrm{Ga}, 40 \%{ }^{71} \mathrm{Ga}\right.$, both with $I=3 / 2$ ). The best fit to the experimental data is obtained by using the following spin-Hamiltonian parameters: $g=2.01 \pm 0.01, \mathrm{~A}\left({ }^{69} \mathrm{Ga}\right)=0.059 \pm 0.002 \mathrm{~cm}^{-1}$, and $\mathrm{A}\left({ }^{71} \mathrm{Ga}\right)$ $=0.0749 \pm 0.002 \mathrm{~cm}^{-1}$. The ODMR curves simulated by using these parameters and assuming involvement of both $\mathrm{Ga}$ isotopes are shown in Fig. 2. To further confirm the validity of this assignment, measurements at two different MW frequencies were performed and are displayed in Figs. 2(a) and 2(b). The agreement between the simulations and the experimental results is excellent, thus justifying the assignments of the defects and reliability of the obtained fitting parameters. ${ }^{12} \mathrm{We}$ note that the defect center with the same parameters was previously detected in the MBE-grown GaNAs and was identified as a Ga-interstitial complex denoted by $\mathrm{Ga}_{i}-\mathrm{D}$. $^{9}$ Based on this similarity, the same $\mathrm{Ga}_{\mathrm{i}}-\mathrm{D}$ label will also be used for the defect discussed in the present study. The modeling has also revealed that, in addition to $\mathrm{Ga}_{\mathrm{i}}-\mathrm{D}$, two signals labeled as $\mathrm{L} 1$ and $\mathrm{L} 2$ in Fig. 2 contribute to the measured ODMR spectra. They originate from two different paramagnetic centers of an effective electron spin $\mathrm{S}=1 / 2$ with $\mathrm{g}=2.010$ and $\mathrm{g}=1.956$ for $\mathrm{L} 1$ and L2, respectively. A lack of a resolved hyperfine structure hinders chemical identification of the corresponding defects which, therefore, will be omitted from further discussion in the paper.

In principle, ODMR studies alone are incapable of determining absolute defect concentrations. However, they yield information on relative defect content in the samples and therefore, allow us to analyze the defect formation in the GaNAsP alloys as a function of the nitrogen and arsenic content. According to the results displayed in Fig. 1(b), the formation of $\mathrm{Ga}_{\mathrm{i}}-\mathrm{D}$ is facilitated by an increase in the $\mathrm{N}$ content above $0.6 \%$. This is in analogy to the behavior of other $\mathrm{Ga}_{\mathrm{i}}-$ related defects in ternary GaNP alloys grown by MBE, where the defect formation was found to be largely promoted by the presence of nitrogen. ${ }^{7}$ This indicates that either an $\mathrm{N}$ atom(s) is directly involved as a part of the $\mathrm{Ga}_{\mathrm{i}}$-related complexes, or $\mathrm{N}$ incorporation provides favorable conditions for 

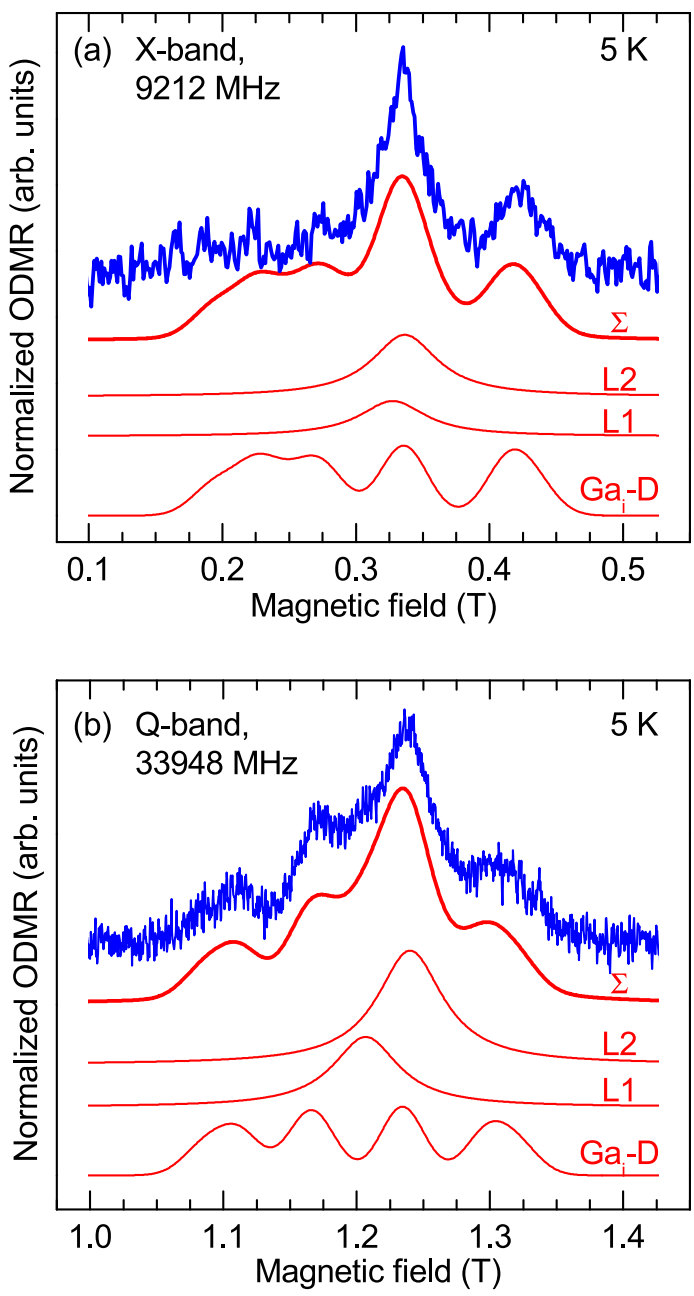

FIG. 2. Representative ODMR spectra measured at $5 \mathrm{~K}$ in (a) $\mathrm{X}$-band and (b) Q-band from the $\mathrm{GaN}_{0.012} \mathrm{As}_{0.05} \mathrm{P}_{0.938}$ epilayer (sample \#12). The uppermost curves in (a) and (b) are the experimental spectra measured by monitoring the total intensity of the PL emissions in the $630-710 \mathrm{~nm}$ spectral range. The simulated ODMR spectra from the three contributing defects are shown by the lowest three curves. The ODMR spectra simulated including the contributions of all there defects are labeled as " $\Sigma$ " and are shown by the thick curves below the experimental spectra for an easy comparison. The applied magnetic field is directed parallel to the [011] crystallographic direction and the ODMR intensity is normalized to the PL intensity. The ODMR signals are isotropic and negative but they are shown as positive for easy viewing.

their formation. The latter can be understood assuming, e.g., that one of driving forces for the formation of interstitials could be local tensile strain induced by the substitution of a large $\mathrm{P}$ atom by a small $\mathrm{N}$ atom. The formation of $\mathrm{Ga}_{\mathrm{i}}$ in the vicinity of $\mathrm{N}$ could then reduce the strain energy making the defect formation energetically favorable. ${ }^{9}$ Consistent with this idea, the most recent first-principle calculations ${ }^{13}$ have concluded that the formation energy for $\mathrm{Ga}_{\mathrm{i}}$ defects is very low and could become even negative due to local bonding effects induced by surrounding $\mathrm{N}$ atoms. This means that these defects are expected to be abundant in dilute nitrides, which further supports our previous conclusions ${ }^{7-9}$ on technological importance of this class of defects.

From Fig. 1, one also notices that incorporation of As in the alloy causes a decrease of the $\mathrm{Ga}_{\mathrm{i}}-\mathrm{D}$ ODMR signal (see Fig. 1(b)), i.e., it has the opposite effect as compared with N. This may mean that the presence of As decreases the local tensile strain which results in an increase in the formation energy and, therefore, a lower concentration of $\mathrm{Ga}_{\mathrm{i}}$. Alternatively, the As-rich conditions may facilitate formation of other defects that are competing with $\mathrm{Ga}_{\mathrm{i}}-\mathrm{D}$ in carrier recombination but cannot be detected by the ODMR technique. The last alternative seems to be more plausible. Indeed, all revealed ODMR signals are negative which means that enhancement of carrier recombination via the involved paramagnetic centers under the magnetic resonance conditions leads to a decrease of the monitored near-band-edge emission. This fact unambiguously proves that the corresponding defects act as efficient recombination centers that compete with the monitored radiative recombination. ${ }^{14}$ Consistently, the overall intensity of the near-band-edge PL decreases with increasing $\mathrm{N}$ content, i.e., under the conditions when the $\mathrm{Ga}_{i}{ }^{-}$ $\mathrm{D}$ defects are effectively formed in the alloy. However, a decrease in the ODMR intensity in the As-containing alloys does not lead to an increase in the PL intensity which in fact is further reduced upon As incorporation. This suggests the formation of other defects that compete with radiative recombination but could not be detected by the ODMR technique (e.g., are not paramagnetic).

Let us now discuss possible local surrounding of the $\mathrm{Ga}_{\mathrm{i}} \mathrm{-D}$ defect. In dilute nitrides, $\mathrm{Ga}_{l}-$ related defects are formed in several configurations which differ by the hyperfine interaction strength and, therefore, local surrounding and/or a partner of the $\mathrm{Ga}_{\mathrm{i}}$ inside the complex. An interstitial atom in the zinc-blende III-V lattice may reside in three high symmetry positions. Two of these positions are of $T_{d}$ symmetry with group-III or group-V atoms in the nearest shell, whereas the third one with the $\mathrm{D}_{3 \mathrm{~d}}$ symmetry corresponds to an interstitial atom surrounded by both group-III and group $\mathrm{V}$ sublattices. Since As and P atoms have different nuclear spins and nuclear magnetic moments, exchanging one of these atoms in the nearest shell of a $\mathrm{Ga}_{i}$ is expected to lead to an observable change in the HF interaction strength. Interestingly, we found that the HF interaction strength remains unaffected by an increase of the As content from 5\% to $18 \%$ (sample \#12 versus sample \#9, see Fig. 1(b)). Moreover, $\mathrm{Ga}_{i}$ with the same HF interaction strength was also observed in GaNAs grown by metal organic chemical vapor deposition (MOCVD). ${ }^{9}$ The observed insensitivity of the HF interaction strength to the group- $\mathrm{V}$ element suggests that neither As nor $P$ are a part of the nearest shell surrounding the $\mathrm{Ga}_{\mathrm{i}}$-atom. This leads to the conclusion that $\mathrm{Ga}_{\mathrm{i}}-\mathrm{D}$ resides at the center of a tetrahedron formed by four group-III atoms, e.g., Ga. Such configuration was also found to be the most favorable from the total energy considerations. ${ }^{13}$ We need to note that the same local surrounding was also concluded for the $\mathrm{Ga}_{\mathrm{i}}-\mathrm{A}$ defect in $\mathrm{Ga}(\mathrm{Al}) \mathrm{NP}^{7}$ and $\mathrm{Ga}(\mathrm{Al}) \mathrm{NAs},{ }^{8}$ which has the strength of hyperfine interaction of $\mathrm{A}\left({ }^{69} \mathrm{Ga}\right)=0.077 \mathrm{~cm}^{-1}$, i.e., larger than that for $\mathrm{Ga}_{\mathrm{i}}-\mathrm{D}$. The reduced strength of the hyperfine interaction may imply that $\mathrm{Ga}_{\mathrm{i}}-\mathrm{D}$ is likely a complex defect containing a $\mathrm{Ga}_{\mathrm{i}}$, where weaker localization of the electron wavefunction on the $\mathrm{Ga}_{\mathrm{i}}$ atom is expected.

Although $\mathrm{Ga}_{i}$-related defects are commonly observed in both MBE and MOVPE grown dilute nitride alloys, different configurations of $\mathrm{Ga}_{i}$ are formed in materials produced by these two growth techniques. The $\mathrm{Ga}_{i}-\mathrm{D}$ configuration seems to be unique to MOCVD-growth, the $\mathrm{Ga}_{i}$-D defect has so far only been found ${ }^{9}$ in the MOCVD-grown GaNAs. On the 
other hand, other $\mathrm{Ga}_{i}$-species such as $\mathrm{Ga}_{i}-\mathrm{A}, \mathrm{Ga}_{i}-\mathrm{B}, \mathrm{Ga}_{i}-\mathrm{C}$, and $\mathrm{Ga}_{i}-\mathrm{E}$ were detected ${ }^{7-9,15}$ in MBE-grown alloys. In principle, there could be several possible reasons for this effect, such as differences in growth temperature, residual contamination, or surface kinetics during the growth. We believe, however, that the growth temperature is not the main factor in the $\mathrm{Ga}_{\mathrm{i}}-\mathrm{D}$ formation, since we have not observed ${ }^{15}$ this defect in GaNP grown by MBE at $590{ }^{\circ} \mathrm{C}$, i.e., at a similar growth temperature as was used during the MOCVD growth. Under the assumption that a residual contaminant is a part of the $\mathrm{Ga}_{i}$-D defect, possible candidates known to be abundant in MOCVD-grown $\mathrm{GaNP}^{16}$ and $\mathrm{Ga}(\mathrm{In}) \mathrm{NAs}{ }^{17,18}$ materials include carbon and hydrogen impurities. In both alloys, concentrations of $\mathrm{H}$ and $\mathrm{C}$ can easily surpass $10^{19} \mathrm{~cm}^{-3}$ and are usually at least one order of magnitude higher than that typical for materials grown by solid source MBE. ${ }^{19,20}$ The fact that only one $\mathrm{Ga}_{i}$ configuration is found in the MOVPEgrown dilute nitrides can be interpreted as an indication that this specific $\mathrm{Ga}_{i}$ configuration has the lowest formation energy in dilute nitrides grown by chemical reactions.

In conclusion, we have conducted a comprehensive study of the point defect formation in the GaNAsP epilayers grown by chemical vapor deposition. It is found that the incorporation of more than $0.6 \%$ of nitrogen facilitates formation of several paramagnetic defects which act as competing recombination centers and are, therefore, harmful to the performance of optoelectronic devices based on the GaNAsP alloy. One of the defects, namely $\mathrm{Ga}_{\mathrm{i}}-\mathrm{D}$, is identified as a complex defect that has a $\mathrm{Ga}$ interstitial atom at its core. Based on the comparison of the deduced spin-Hamiltonian parameters for $\mathrm{Ga}_{\mathrm{i}}-\mathrm{D}$ with those known for other $\mathrm{Ga}_{\mathrm{i}}$-related defects in ternary GaNP and GaNAs alloys, the $\mathrm{Ga}_{\mathrm{i}}-\mathrm{D}$ configuration is concluded to be the dominant configuration of the $\mathrm{Ga}_{\mathrm{i}}$-related defects in MOVPE-grown dilute nitrides. It is also shown that $\mathrm{Ga}_{\mathrm{i}}-\mathrm{D}$ involves a $\mathrm{Ga}_{\mathrm{i}}$ atom that is most likely surrounded by the group-III sublattice.

Financial support by the Swedish Research Council (Grant \# 621-2010-3815), the Swedish Institute via Visby Programme, and Linköping Linnaeus Initiative for Novel
Functional Materials (LiLI-NFM) supported by the Swedish Research Council (contract number 2008-6582) is highly appreciated.

${ }^{1}$ D. Linag and J. E. Bowers, Nature Photon. 4, 511 (2010).

${ }^{2}$ For a review, see in Physics and Applications of Dilute Nitrides, edited by I. A. Buyanova and W. M. Chen (Taylor \& Francis, London, 2004).

${ }^{3}$ For a review, see Dilute III-V Nitride Semiconductors and Material Systems, Springer Series in Material Science, Vol. 105, edited by A. Erol (Springer, Berlin, 2008).

${ }^{4}$ W. Shan, W. Walukiewicz, K. M. Yu, J. Wu, J. W. Ager III, E. E. Haller, H. P. Xin, and C. W. Tu, Appl. Phys. Lett. 76, 3251 (2000).

${ }^{5}$ I. A. Buyanova, G. Pozina, J. P. Bergman, W. M. Chen, H. P. Xin, and C. W. Tu, Appl. Phys. Lett. 81, 52 (2002).

${ }^{6}$ E. R. Weber, Physica B: Cond. Matter 340-342, 1 (2003).

${ }^{7}$ N. Q. Thinh, I. P. Vorona, I. A. Buyanova, W. M. Chen, S. Limpijumnong, S. B. Zhang, Y. G. Hong, H. P. Xin, C. W. Tu, A. Utsumi, Y. Furukawa, S. Moon, A. Wakahara, and H. Yonezu, Phys. Rev. B 71, 125209 (2005).

${ }^{8}$ I. P. Vorona, T. Mchedlidze, D. Dagnelund, I. A. Buyanova, W. M. Chen, and K. Köhler, Phys. Rev. B 73, 125204 (2006).

${ }^{9}$ X. J. Wang, Y. Puttisong, C. W. Tu, A. J. Ptak, V. K. Kalevich, A. Y. Egorov, L. Geelhaar, H. Riechert, W. M. Chen, and I. A. Buyanova, Appl. Phys. Lett. 95, 241904 (2009).

${ }^{10}$ A. Yu. Egorov, N. V. Kryzhanovskaya, and M. S. Sobolev, Semiconductors 45, 1164 (2011).

${ }^{11}$ I. A. Buyanova, G. Yu. Rudko, W. M. Chen, H. P. Xin, and C. W. Tu, Appl. Phys. Lett. 80, 1740 (2002).

${ }^{12}$ Weaker intensities of the experimental ODMR lines at low fields as compared with that in the simulated spectra are because of modifications of recombination rates by mixing of states which become important at the low fields but were not included in the simulations.

${ }^{13}$ P. Laukkanen, M. P. J. Punkkinen, J. Puustinen, H. Levämäki, M. Tuominen, K. Schulte, J. Dahl, J. Lång, H. Zhang, M. Kuzmin, K. Palotas, B. Johansson, L. Vitos, M. Guina, and K. Kokko, Phys. Rev. B 86, 195205 (2012).

${ }^{14}$ W. M. Chen, Thin Solid Films 364, 45 (2000).

${ }^{15}$ D. Dagnelund, I. A. Buyanova, X. J. Wang, W. M. Chen, A. Utsumi, Y. Furukawa, A. Wakahara, and H. Yonezu, J. Appl. Phys. 103, 063519 (2008).

${ }^{16}$ J. F. Geisz, R. C. Reedy, B. M. Keyes, and W. K. Metzger, J. Cryst. Growth 259, 223 (2003).

${ }^{17}$ A. Moto, M. Takahashi, and S. Takagishi, J. Cryst. Growth 221, 485 (2000).

${ }^{18}$ S. Kurtz, J. F. Geisz, B. M. Keyes, W. K. Metzger, D. J. Friedman, J. M. Olson, and A. J. Ptak, Appl. Phys. Lett. 82, 2634 (2003).

${ }^{19}$ A. J. Ptak, S. W. Johnston, S. Kurtz, D. J. Friedman, and W. K. Metzger, J. Cryst. Growth 251, 392 (2003).

${ }^{20}$ D. Dagnelund, I. P. Vorona, G. Nosenko, X. J. Wang, C. W. Tu, H. Yonezu, A. Polimeni, M. Capizzi, W. M. Chen, and I. A Buyanova, J. Appl. Phys. 111, 023501 (2012). 